\title{
Invasión cardiaca de carcinoma sarcomatoide pulmonar a través de las venas pulmonares
}

\section{Carolina Rodríguez $^{\mathrm{a}, *}$, Fernando Fortich ${ }^{\mathrm{b}, \mathrm{c}}$ y Erwin D. Quintero ${ }^{\mathrm{d}}$}

\author{
a Departamento de Anestesiología, Universidad de Antioquia, Medellín, Colombia \\ b Departamento de Cardiología, Hospital San Vicente Fundación, Medellín, Colombia \\ c Departamento de Cardiología, Universidad de Antioquia, Medellín, Colombia \\ d Departamento de Radiología, Universidad de Antioquia, Medellín, Colombia
}

Recibido el 4 de julio de 2013; aceptado el 7 de mayo de 2014

Disponible en Internet el 2 de octubre de 2014

\section{PALABRAS CLAVE}

Tumores;

Venas;

Diagnóstico

\section{KEYWORDS}

Tumors;

Veins;

Diagnosis
Resumen Los tumores cardiacos son una entidad poco común; pueden ser resultado de metástasis o tumor primario originado en cualquiera de las cavidades cardiacas, o pueden llegar al corazón por invasión directa a través de las venas pulmonares de un tumor originario del pulmón, fenómeno que es aún más raro de acuerdo con lo reportado en la literatura hasta el día de hoy.

Se publica el caso de una paciente que comenzó con deterioro de su clase funcional y con síntomas respiratorios; a quien con base en imágenes diagnósticas, se le detectó carcinoma sarcomatoide pulmonar que invadía el tracto de salida del ventrículo izquierdo a través de las venas pulmonares.

Se discuten la incidencia de estos tumores, la sintomatología, las ayudas diagnósticas disponibles y las opciones de tratamiento.

(c) 2013 Sociedad Colombiana de Cardiología y Cirugía Cardiovascular. Publicado por Elsevier España, S.L.U. Todos los derechos reservados.

\section{Cardiac invasion of pulmonary sarcomatoid carcinoma through the pulmonary veins}

Abstract Metastatic cardiac tumors are more common than primary ones; they may extend by contiguity through the pulmonary veins to the heart chambers. Although this is an unusual event, it has been reported with different types of tumors.

We report the case of a patient who started with deterioration of her functional class and with respiratory symptoms. Diagnostic imaging showed a pulmonary sarcomatoid carcinoma invading the left ventricular outflow tract through the pulmonary vein.

\footnotetext{
* Autor para correspondencia.

Correo electrónico: carolinarodm@gmail.com (C. Rodríguez).
} 
We discuss the impact of these tumors, symptoms, diagnostic aid that we have, as well as the treatment options.

(c) 2013 Sociedad Colombiana de Cardiología y Cirugía Cardiovascular. Published by Elsevier España, S.L.U. All rights reserved.

\section{Introducción}

El tumor cardiaco primario más común es el mixoma auricular ${ }^{1}$; aun así las metástasis a corazón, de sitios como pulmón y mama, son de veinte a cuarenta veces más comunes que los tumores primarios ${ }^{2}$. La extensión al corazón se puede dar a través de cuatro vías: directa (consecuencia de la contigüidad o infiltración directa), hemática, linfática o a través de la vena cava o las venas pulmonares ${ }^{3}$; esta última es menos común que las demás vías.

Se presenta el caso de una paciente con un tumor pulmonar que se extendió a las cavidades cardiacas a través de las venas pulmonares, cuyo diagnóstico histopatológico confirmó un carcinoma sarcomatoide.

\section{Caso}

Paciente de género femenino, de 61 años de edad, con antecedente de tabaquismo pasivo importante más no activo, sin otros antecedentes de importancia y clase funcional NYHA I hasta tres meses antes de su ingreso en la institución. Consultó por tos seca, diaforesis, astenia, adinamia y pérdida objetiva de $5 \mathrm{~kg}$. Fue hospitalizada en dos ocasiones en otras instituciones, donde diagnosticaron neumonía adquirida en la comunidad, para lo cual recibió tratamiento antibiótico y tuvo mejoría clínica sin resolución completa de su sintomatología. Por persistencia de los síntomas respiratorios consultó nuevamente y en esta ocasión le fue realizada una radiografía de tórax donde evidenciaron imagen radioopaca en base pulmonar derecha y sugirieron cita por consulta externa.

Ingresó en nuestra institución por empeoramiento de la disnea, tos seca persistente, pobre tolerancia al decúbito, adinamia marcada y dolor pleurítico; palidez, edema grado Il en miembros inferiores e hipoventilación en campo pulmonar derecho con sibilancias espiratorias difusas asociadas. Se documentó $\mathrm{Hb}: 6,5 \mathrm{~g} / \mathrm{dL}$ (aportó resultados ambulatoria de 9,1 meses atrás) y se decidió trasfundir. Se solicitó tomografía axial computarizada de tórax contrastado (fig. 1) donde se evidenciaron múltiples ganglios linfáticos paraesofágicos, perivasculares y en la ventana aortopulmonar, adenopatías para y pretraqueales derechas. A nivel del bronquio fuente derecho se observó lesión intraluminal de $11 \times 6,5 \mathrm{~mm}$; se continuó con una masa de densidad heterogénea, que ocupaba el lóbulo medio e inferior del pulmón derecho, con hipodensidad central, la cual medía $94 \times 109 \times 100 \mathrm{~mm}$ y comprometía la arteria pulmonar interlobar derecha ocasionando efecto compresivo sobre la cava, la aurícula derecha, el bronquio para el lóbulo superior derecho y el diafragma derecho. Adicionalmente se evidenció una imagen hipodensa irregular a nivel de la aurícula izquierda que fue leída como posible trombo. Se decidió complementar el estudio de este hallazgo con ecocardiografía transtorácica cuyo reporte informó masa compatible con mixoma en la aurícula izquierda de $13 \mathrm{~cm} \times 8 \mathrm{~cm} \times 1,4 \mathrm{~cm}$ con insuficiencia mitral severa por protrusión de la masa al ventrículo.

Se revisaron las imágenes antes de optar por una conducta quirúrgica del mixoma y se solicitó ecocardiografía transesofágica en donde se reportaron múltiples masas gigantes ecodensas, una de ellas en el tracto de salida del ventrículo izquierdo. Se solicitó resonancia magnética cardiaca para determinar si se trataba de la misma masa pulmonar o de metástasis (fig. 2). Las imágenes mostraron gran masa pulmonar neoplásica con invasión vascular mediastinal que invadía las venas pulmonares y con extensión a la aurícula izquierda y a la válvula mitral; adicionalmente se observó un nódulo adherido a la valva anterior de la mitral que causaba obstrucción dinámica parcial al tracto de salida del ventrículo izquierdo.

En el transcurso de la hospitalización y por su condición respiratoria limítrofe, se decidió hacer biopsia percutánea de la lesión pulmonar dirigida por tomografía axial computarizada. El informe preliminar por patología determinó neoplasia pulmonar maligna indiferenciada que se decidió aclarar con coloraciones de inmunohistoquímica.

Por el pronóstico y el grado de invasión fue dada de alta con oxígeno domiciliario, cita con Oncología, anticoagulación y medidas paliativas de manejo ambulatorio.

Cuatro días después del alta, reingresó por disnea limitante; se hallaron signos de dificultad respiratoria, por lo que inició manejo con broncodilatadores con respuesta inicial. Sin embargo, horas después de este último ingreso se tornó desorientada y con inestabilidad hemodinámica. Se explicó a la familia y se decidió limitar esfuerzos terapéuticos. Falleció siete horas después de este ingreso.

El reporte de patología confirmó carcinoma sarcomatoide.

\section{Discusión}

Las neoplasias intracardiacas pueden dividirse en primarias o secundarias según sea su origen, y benignas o malignas de acuerdo con sus características histopatológicas. La invasión de un carcinoma sarcomatoide a través de las venas pulmonares, la cual tiene una incidencia de 0,1 a 0,4\% en cuanto a tumores pulmonares ${ }^{4}$, es un evento muy raro. Hasta ahora, Weng et al. habían publicado el único reporte en la literatura. 


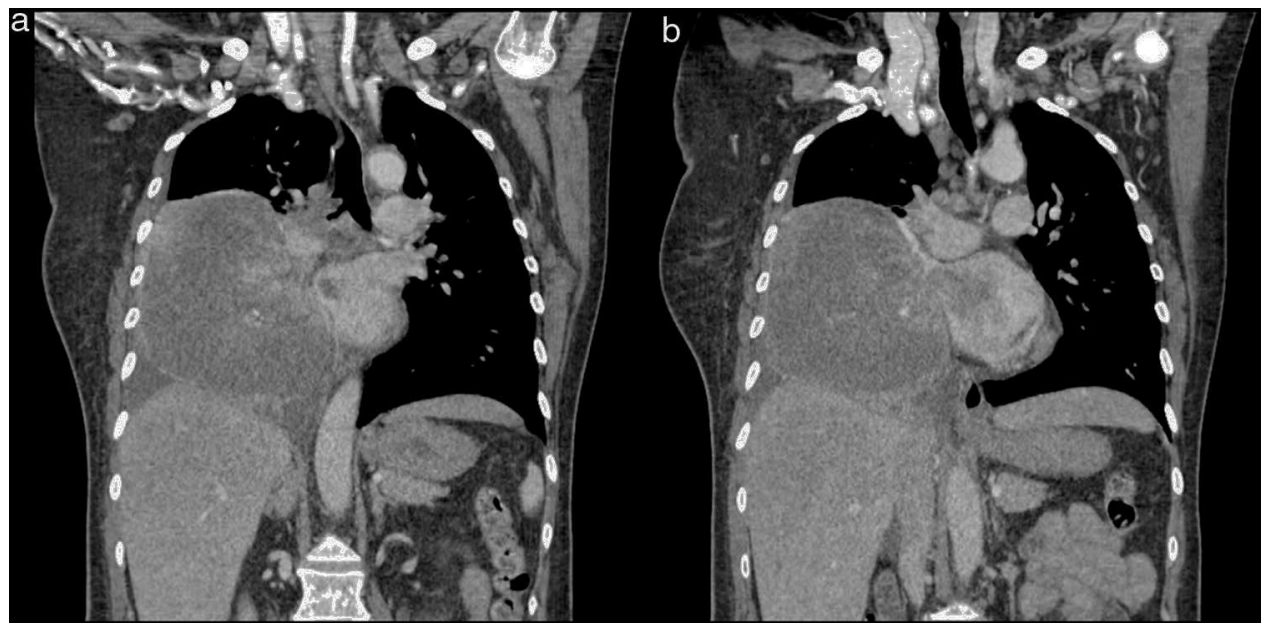

Figura 1 Reconstrucciones coronales que muestran lesión con densidad de tejidos blandos, de tipo infiltrativo de comportamiento agresivo, que compromete el bronquio (izquierda) y el lóbulo medio e inferior derecho, con áreas de realce heterogéneo, efecto de masa en las estructuras hiliares ipsilaterales; desvía la tráquea y en sentido contrario deprime el diafragma. Se observa, además, derrame pleural derecho, linfadenopatía descrita, imagen hipodensa de defecto de llenado del medio de contraste en la aurícula izquierda, interpretado como trombo (derecha).

Se caracterizan por aumentar rápidamente de tamaño y por poseer características histopatológicas bifásicas: sarcomatoides y epiteliales. La supervivencia es similar a la de los tumores no sarcomatoides y el pronóstico depende de la localización, el tamaño, la profundidad de la invasión y la condición clínica del paciente ${ }^{4}$.

Existen otros tumores pulmonares de los cuales hay reportes de invasión cardiaca a través de las venas pulmonares, entre ellos el carcinoma escamocelular, el adenocarcinoma, el carcinoma adenoescamoso, el carcinoma mucoepidermoide, el tumor carcinoide atípico, el carcinoma de células grandes y de células pequeñas, el leiomiosarcoma y el condrosarcoma, incluso en algunos reportes como el que se publica, tienden a confundirse con un tumor primario cardiaco: mixoma o trombo ${ }^{4}$.

La sintomatología es variable; en este caso la paciente comenzó con síntomas de origen pulmonar e incidentalmente se encontró la masa cardiaca que en ningún momento ocasionó síntomas cardiovasculares a pesar de su insuficiencia mitral asociada y la protrusión de la masa, casi hasta obstruir el tracto de salida del ventrículo izquierdo. Los tumores pulmonares generalmente se manifiestan por crecimiento local en cuyo caso ocasionan hemoptisis o disnea, o por invasión de estructuras adyacentes causando dolor pleurítico, síndrome de vena cava superior, parálisis del frénico o diseminación linfática con obstrucción traqueal o esofágica ${ }^{5}$. La arritmia es otra forma de presentación del compromiso cardiaco como describen Brahmbhatt et al. en su reporte de caso ${ }^{5}$. Es poco común encontrar un soplo como signo clínico de tumor cardiaco y cuando existe generalmente es sistólico dada la pobre movilidad de la masa durante la diástole del ciclo cardiaco ${ }^{6}$.

Según la sintomatología cardiovascular, las ayudas diagnósticas comenzarán por lo básico. La radiografía de tórax orienta el origen de la patología; en la paciente del caso por ejemplo, se demostró una densidad derecha poco caracterizable en los rayos $X$, por la complejidad del abordaje diagnóstico. Entre tanto, la tomografía ofrece una caracterización estructural adecuada que permite valorar el origen de la lesión: si es primaria cardiaca o demuestra signos de invasión secundaria del corazón a partir de neoplasias mediastinales, si se definen los planos de clivaje, las líneas grasas, la separación de las membranas, por ejemplo en el derrame, o si, por el contrario se evidencia la invasión cardiaca; la forma más común de compromiso cardiaco metastásico al pericardio es el taponamiento y presenta hallazgos claramente identificables en las imágenes tomográficas. La resolución tisular de la tomografía, donde solamente se valoran la densidad y el realce con el medio de contraste, a veces como en este caso, es insuficiente para determinar el origen de la lesión cardiaca. La ecografía transtorácica es sensible pero poco específica, con limitantes como la contextura corporal del paciente y el pequeño campo de visión. Aunque la ecocardiografía transesofágica es invasiva, es una herramienta que permite identificar las diferentes válvulas, determinar si hay compromiso de las mismas, establecer la función ventricular, así como el estado del pericardio y su posible compromiso, además de masas intracavitarias que interfieran el flujo sanguíneo; adicionalmente es factible evaluar el tamaño y la extensión del tumor. La resonancia magnética, de otra parte, tiene la mejor diferenciación tisular de los tejidos blandos, puede aclarar el contenido graso, por ejemplo en un lipoma, necrosis de los tumores en las secuencias anatómicas en T1y T2, y es sensible a los productos paramagnéticos y al movimiento de las moléculas con secuencias de eco de gradiente. En el análisis de la imagen debe tenerse claro si el comportamiento tumoral es benigno o maligno, como en el realce con el medio de contraste, la invasión tumoral, o un tamaño mayor a $5 \mathrm{~cm}$, lo que favorece el diagnóstico de tumor maligno; además, la mejor resolución de contraste que se tiene con la resonancia en comparación con la ecografía y el amplio campo de visión (FOV: field of view), permite caracterizar esta lesión contactándola con la masa pulmonar y demostrando el mismo comportamiento por imagen en relación con compromiso metastásico, lo cual ayuda a diferenciarla de un trombo que es mucho más frecuente que la invasión tumoral cardiaca y de un mixoma que sería otro diagnóstico diferencial importante. 

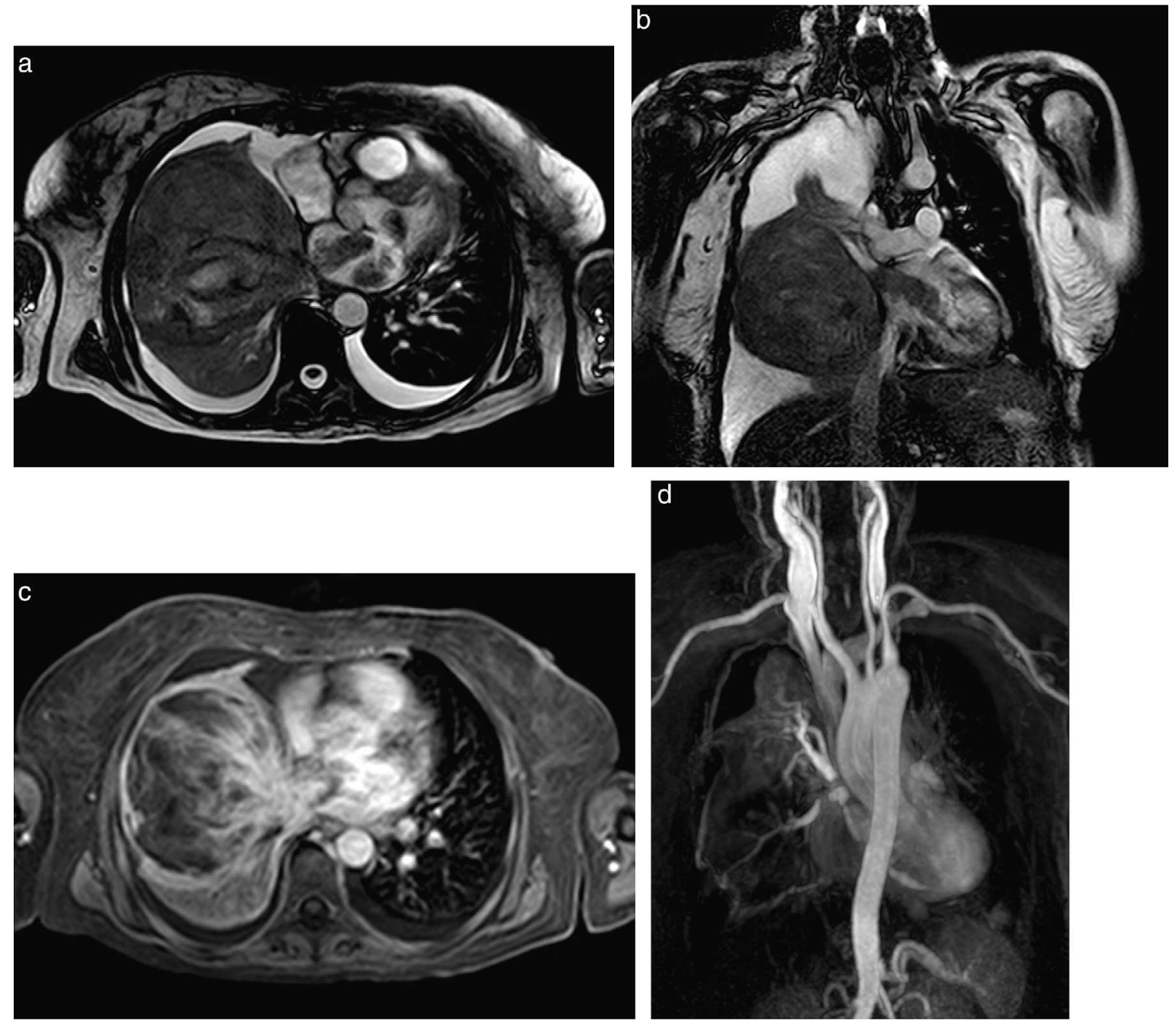

Figura 2 Imágenes de resonancia magnética en secuencias balanceadas (a) que muestran derrame pleural bilateral, masa pulmonar derecha de señal heterogénea predominantemente hipointensa al líquido, hiperintensa al parénquima pulmonar normal contralateral, con zonas centrales hiperintensas por necrosis, con dos imágenes nodulares en la aurícula izquierda de comportamiento magnético idéntico a la lesión y otra lesión en el tracto de salida del ventrículo izquierdo, que se proyecta a través de la válvula mitral (b) que presenta realce con el medio de contraste similar al realce periférico de la masa pulmonar (c) por mneoplásica extensión cardiaca a través de las venas pulmonares. Disminución de la intensidad de señal atrial izquierda en la reconstrucción de máxima intensidad de la secuencia de angio-rm por efecto de las masas descritas (d).

La valoración dinámica por resonancia permite evaluar el impacto funcional cardiaco en tiempo real así como la ecocardiografía, con la ventaja de tener un campo de visión global del tórax que se hace esencial en alteraciones complejas, como en este caso, donde se observa claramente la invasión secundaria. Cada vez esta modalidad de imagen está más expandida y asequible, puesto que mejora el rendimiento diagnóstico y la planeación quirúrgica en los pacientes, sin la limitación de administración de radiación ionizante ${ }^{7,8}$.

El manejo dependerá de las características del tumor, el grado de invasión, el compromiso de los ganglios linfáticos y el pronóstico de la enfermedad. Fukuse et al. reportaron 930 casos de cáncer de células pequeñas de los cuales 47 eran invasores y de ellos 14 invadían la aurícula izquierda; y fueron llevados a cirugía para resección. La supervivencia a tres años fue del 17\%, menor en los casos de invasión aurícular izquierda9. Una opción antes de llegar a la resección quirúrgica del tumor es realizar quimioterapia con el objetivo de disminuir el tamaño del mismo para posteriormente considerar estrategias quirúrgicas definitivas como la neumonectomía y la resección de la aurícula en bloque ${ }^{10}$. Solo en casos muy seleccionados se ha indicado la resección de metástasis cardiacas con el objetivo de paliar síntomas y prolongar la vida ${ }^{11}$.

En el caso expuesto, debido al tamaño del tumor y la condición avanzada de la paciente la única alternativa posible fue la estabilización de su estado para considerar un manejo paliativo posterior ya que era poco factible una respuesta a la quimioterapia; además en vista del gran tamaño y su compromiso pulmonar y cardiaco, la cirugía, como se relató previamente, implicaría una resección amplia con pocas posibilidades de tolerancia. Finalmente, se dio espera a una mejor condición clínica pero la muerte temprana de la paciente no dio tiempo para intentar las demás opciones. 


\section{Conclusión}

La invasión cardiaca de un tumor pulmonar a través de las venas pulmonares existe y se debe sospechar en pacientes con tumores de rápido crecimiento, en quienes la sintomatología puede ser tanto del sistema pulmonar como del cardiovascular. Las posibilidades diagnósticas siempre empezarán por lo básico y la ecocardiografía transesofágica tendrá un papel importante en la evaluación de la masa, sus repercusiones, el compromiso de las diferentes estructuras del corazón y la función del mismo, aun así se cuenta con la resonancia magnética cardiaca que permitirá una mejor diferenciación de los tejidos y al mismo tiempo evaluará la función cardiaca. El tratamiento es complejo ya que lo ideal siempre será disminuir el tamaño con quimioterapia para poder proceder a resección quirúrgica de las masas, y de las estructuras afectadas siempre y cuando la condición clínica y el pronóstico del paciente lo permitan.

\section{Conflicto de intereses}

Los autores declaran no tener ningún conflicto de intereses.

\section{Bibliografía}

1. Nakanishi K, Nagaie T, Tanaka J, Ando H. Concomitant surgery of a left atrial myxoma and a pulmonary carcinoma. Gen Thorac Cardiovasc Surg. 2007;55:325-7.
2. Dodos F, Schneider T, Fries J, Hopper U. Benign cardiac tumor in a patient with lung malignancy. Clin Res Cardiol. 2007;96:628-31.

3. Kadappu K, Rajaratnam R, Kachwalla H, Nguyen P. Lung cancer mimicking left atrial mass. Postgrad med J. 2008;84: $386-7$.

4. Ng SW, Lim CH, Wang CW, Chua YL. Primary pulmonary sarcomatoid carcinoma with intracardiac extension. Jpn J Thorac Cardiovasc Surg. 2006;54:416-9.

5. Brahmbhatt R, Dorsogna Ll, Cooke P. Primary lung cancer involving mediastinum and mimicking left atrial myxoma: A case report and review of literature. Heart Lung and Circulation. 2005; 14:48-50.

6. Brandt RR, Rubin J, Reeder GS. Intracardiac extension of a lung tumor causing left ventricular inflow obstruction. J Am Soc Echocardiogr. 1995;8:930-3.

7. Buckley O, Madan R, Kwong R, Rybicki F, Hunsaker A. Cardiac masses. Part 2: Key imaging features for diagnosis and surgical planning. AJR. 2011;197:W842-51.

8. Sparrow P, Kurian JB, Jones TR, Sivananthan TR, Mohan U. MR imaging of cardiac tumors. Radio Graphics. 2005;25:1255-76.

9. Fukuse $\mathrm{T}$, Wada $\mathrm{H}$, Hitomi S. Extended operation for non small cell lung cancer invading great vessels and left atrium. Eur J Cardio Thorac Surg. 1997;11:664-9.

10. Labib SB, Schick Jr EC, Isner JM. Obstruction of right ventricular outflow tract caused by intracavitary metastatic disease: analysis of 14 cases. J Am Coll Cardiol. 1992;19: 1664-8.

11. Messner G, Harting MT, Russo P, Gregoric ID, Mukhopadhyay M, Flamm SD, et al. Surgical management of metastatic melanoma to the ventricle. Tex Heart Inst J. 2003;30:218-20. 